Check for updates

Cite this: RSC Adv., 2019, 9, 3558

Received 3rd December 2018

Accepted 14th January 2019

DOI: $10.1039 / c 8 r a 09934 k$

rsc.li/rsc-advances

\title{
Obtaining high mechanical performance silk fibers by feeding purified carbon nanotube/ lignosulfonate composite to silkworms $\uparrow$
}

\author{
Hao Xu, ${ }^{a}$ Wenhui $Y_{i}{ }^{\star a}$ Dongfan Li, ${ }^{b}$ Ping Zhang, (D) ${ }^{a}$ Sweejiang Yoo, ${ }^{a}$ Lei Bai, ${ }^{a}$ Jin $\mathrm{Hou}^{\mathrm{c}}$ \\ and Xun Hou ${ }^{a}$
}

Silkworm fibers have attracted widespread attention for their superb glossy texture and promising mechanical performance. The mechanical properties can be reinforced with carbon nanofillers, particularly carbon nanotubes (CNTs), depending on the CNT content in the silk fibers. In order to increase the CNT content, lignosulfonate (LGS) was used as a surfactant to ameliorate the CNT solubility, dispersibility, and biocompatibility. The resulting CNT/LGS nano-composite was further processed through an additional purification method to remove excess surfactant and enhance the CNT/LGS ratio. Then the purified biocompatible single and multiple-walled CNTs were fed to silkworms, leading to a large CNT content in the resulting silk fibers. Reinforced silk fibers were produced with a mechanical strength as high as $1.07 \mathrm{GPa}$ and a strain of $16.8 \%$. The toughness modulus is 1.69 times than that of the unpurified group. The CNT-embedded silk fibers were characterized via Raman spectrometry and thermogravimetric analysis (TGA), demonstrating that the CNT content in the silk fibers increased 1.5fold in comparison to the unpurified group. The increased CNT content not only contributed to the selfassembly into buffering knots of silk fibers, but it also enhanced the conductivity of graphitized silk. Our coating and purification strategies provide a potential facile way to obtain natural silk fibers with high mechanical performance.

\section{Introduction}

Natural silk fibers have drawn much attention in biology, physical chemistry, materials science, and other areas, because of their ecofriendly sustainability and promising mechanical properties (e.g., strength and stretchable nature). ${ }^{1-4}$ In particular, silk fibers produced by the mulberry silkworm have been widely used in tissue engineering, ${ }^{5,6}$ controlled drug release, ${ }^{7,8}$ wound dressing, ${ }^{9}$ silk hydrogels, ${ }^{10}$ bio-photonics, ${ }^{11,12}$ wearable human-motion sensors and electrochemical diagnosis, ${ }^{13,14}$ due to their lustrous texture, biodegradability, outstanding toughness, and excellent biocompatibility. Various methods have been developed to improve the mechanical, electrical, and optical properties of silk fibers, such as filling them with carbon-based nanomaterials ${ }^{15-17}$ functionalization with

${ }^{a}$ Key Laboratory for Information Photonic Technology of Shaanxi Province, Key Laboratory for Physical Electronics and Devices of the Ministry of Education, School of Electronics and Information Engineering, Xi'an Jiaotong University, Xi'an 710049, P. R. China. E-mail: yiwenhui@mail.xjtu.edu.cn

${ }^{b}$ Frontier Institute of Science and Technology, Xi'an Jiaotong University, Xi'an 710054 , P. R. China

${ }^{c}$ Department of Pharmacology, School of Basic Medical Sciences, Xi'an Medical University, Xi'an 710021, Shaanxi, People's Republic of China

$\dagger$ Electronic supplementary information (ESI) available. See DOI: 10.1039/c8ra09934k fluorescent dyes ${ }^{18,19}$ and transgenic engineering. ${ }^{20,21}$ For instance, Tansil et al. ${ }^{18,19}$ used fluorescent dyes as nanofillers to obtain colorful functionalized silk fibers. Carbon nanodots ${ }^{22}$ can be supposed to be a promising substitute for those fluorescent dyes, due to their intense photoluminescence, low toxicity, and high aqueous solubility. In addition, inherently reinforced fibers have been obtained through directly feeding artificial additives to silkworms or spiders. ${ }^{15-17}$

Single- or multiple-walled carbon nanotubes (SWCNTs/ MWCNTs) have been widely used in transistors, ${ }^{23,24}$ batteries, ${ }^{25}$ and reinforcing agents, ${ }^{26,27}$ due to their excellent electrical, mechanical and catalytic properties. By filling CNTs into silk fibers, the nanotubes with structural translational symmetry and structural flexibility endow the fibers with improved electrical and mechanical properties. ${ }^{28-30}$ The carbon nanomaterials can be assembled on silk fibroin ex situ through dry/wet-spinning ${ }^{31,32}$ or electrospinning ${ }^{33}$ a mixed solution. Alternatively, by feeding silkworms with mulberry leaves sprayed with single-wall carbon nanotubes or graphene dispersions, silk fibers with in situ developed mechanical properties can be obtained..$^{15}$ It is especially desirable to enhance the CNT/surfactant ratio in any artificial additives, since excess surfactant will block CNTs from entering into the silk fibers. Lignosulfonate (LGS) is used as the surfactant to coat the CNTs because it is a natural product with stable 
hydrophilic functionality and biocompatibility. ${ }^{17}$ The relationship between silk protein sequences and mechanical properties has been reported before. ${ }^{34-36}$ The CNT content in silk fibers can lead to protein reorganization and subsequently afford tunable mechanical properties. ${ }^{15}$ In order to enhance the mechanical properties of silk fibers, it is highly desirable to increase the CNT content in the silk fibers, which can be realized by removing excess LGS coating. Currently, van der Waals forces or $\pi-\pi^{*}$ stacking is used for the non-covalent wrapping of lignosulfonate on CNTs, ${ }^{37,38}$ during the preparation of solutions for feeding silkworms. However, there has been no discussion of the impact of the CNT/LGS ratio on the CNT content in silk fibers. In this work, we designed a purification process to remove excess LGS, which can enhance the CNT/LGS ratio in artificial additives. Then, we obtained high mechanical and electrical performance fibers through feeding the purified artificial additives to Bombyx mori silkworms, which have been domesticated over thousands of years. A qualitative analysis of mechanical strength enhancement and the CNT content in the silk fibers was done using TGA and Raman spectroscopy, respectively. The mechanism of the mechanical strength and electrical conductivity enhancement was carefully studied and well addressed.

\section{Materials and methods}

\section{Materials}

Bombyx mori larvae, a silkworm strain resulting from the hybridization of Qiufeng and Baiyu, were cultivated in a homebuilt hatcher in a laboratory at Xi'an Jiaotong University, China. Fresh mulberry leaves were obtained from the Qinyuan mulberry leaf ecological garden (Shaanxi province, China). A purification installation, including a Jinteng diaphragm pump, a conical bottle of chemical glass, and vacuum filtration accessories, was established in our lab and organic filter paper of $0.2 \mu \mathrm{m}$ was purchased from Tianjin JinTeng Experimental Equipment Co., Ltd. (Tianjin, China). SWCNTs and MWCNTs, grown via chemical vapor deposition and with purity of more than $95 \%$, were purchased from the Chengdu Institute of Organic Chemistry, Chinese Academy of Sciences. The outer diameters of the single- and multi-walled carbon nanotubes were respectively in the ranges of 1-2 nm and 10-20 nm, and the lengths were all in the range of 10-30 $\mu \mathrm{m}$. Calcium lignosulfonate (surface active agent with purity of $96 \%$ ), anhydrous sodium carbonate (analytical reagent with purity of $99.5 \%$ ), and concentrated nitric acid (analytical reagent with purity of 65\%) were purchased from Shanghai Aladdin Bio-Chem Technology Co., LTD (Shanghai, China).

\section{Preparation and purification of silkworm diets}

$0.2 \mathrm{~g}$ duplicate samples of SWCNTs were each mixed with $5 \mathrm{~g}$ of LGS, and then $100 \mathrm{~mL}$ of deionized (DI) water was added to each mixed sample. Subsequently, these two samples were ultrasonicated for $120 \mathrm{~min}$ and then centrifuged at $12000 \mathrm{rpm}$ to generate separate batches of coated CNTs. Similarly, for MWCNT samples, $0.2 \mathrm{~g}$ duplicate samples of MWCNTs were treated in the same manner. Another group containing only $5 \mathrm{~g}$ of LGS served as the control feeding group. To further obtain a highly-concentrated CNT solution, excess LGS was removed through $0.2 \mu \mathrm{m}$ filters multiple times. Effective purification was judged to have been carried out when the dark yellow filtrate gradually became colorless. TGA was used to evaluate the pure/ impure CNT ratio. Detailed information is provided in Fig. $\mathrm{S} 1 \dagger$ and Table 1. The obtained solutions were prepared as novel feeding additives and uniformly distributed on the surfaces of mulberry leaves to feed the silkworms, as shown in Fig. S2. $\dagger$ In addition, a rearing-box $(50 \mathrm{~cm} \times 30 \mathrm{~cm} \times 30 \mathrm{~cm})$ was designed to maintain the temperature at $25 \pm 2{ }^{\circ} \mathrm{C}$ and the relative humidity at $65 \pm 5 \%$. Using this rearing-box, a total of 50 silkworms, randomly divided into five groups in the final phase of the fourth instar, were fed these five additives: purified and unpurified SWCNTs, purified and unpurified MWCNTs, and the control additive.

\section{Preparation of degummed silk samples}

Before degumming, the silkworm cocoons were immerged in DI water at $60{ }^{\circ} \mathrm{C}$ for $30 \mathrm{~min}$ to clean them, and then they were dried in an oven. Next, the cocoons were boiled in a solution of $\mathrm{Na}_{2} \mathrm{CO}_{3}$ at $\mathrm{pH}=10$ for $40 \mathrm{~min}$ and were rinsed more than three times with DI water to make sure that the sericin glue was completely removed. In addition, the degummed silk fibers (SFs) were uniformly collected and then dried in an oven at $70{ }^{\circ} \mathrm{C}$ for 8 hours. After degumming, the collected SFs were placed in an oven at $40^{\circ} \mathrm{C}$ until the silk samples were analyzed.

\section{Field emission scanning electron microscopy}

Small pieces of pristine SF and single SFs at fracture were respectively attached to silicon chips with conductive glue before observation using FESEM; the samples were then were sputtered with Pt to a thickness of $5 \mathrm{~nm}$. After that, the morphologies of the SFs were observed via field emission scanning electron microscopy (FE-SEM) (JEOL 7800F, Japan) with a voltage of $3.0 \mathrm{kV}$ and at two matched magnifications of $1 \mathrm{k}$ and $2 \mathrm{k}$ at a WD of $6 \mathrm{~mm}$.

Table 1 SWCNT and MWCNT content in the feeding additives

\begin{tabular}{lllll}
\hline Additive name & SWCNT [\%] & MWCNT [\%] & LGS [\%] & Treatment method \\
\hline SWCNT1@LGS & 33.44 & - & 66.55 & Purified \\
SWCNT2@LGS & 0.32 & - & 99.67 & Unpurified \\
MWCNT1@LGS & - & 70.47 & 29.52 & Purified \\
MWCNT2@LGS & - & 7.93 & 92.06 & Unpurified
\end{tabular}




\section{Thermogravimetric analysis}

The TGA curves of degummed SFs were measured using a thermogravimetric instrument (NETZSCH STA 449C, Germany) over a temperature range from 25 to $1200{ }^{\circ} \mathrm{C}$ using a heating rate of $5^{\circ} \mathrm{C} \mathrm{min}^{-1}$ and nitrogen flow rate at 20 $\mathrm{mL} \min ^{-1}$ to quantitatively determine the amount of CNTs embedded in the SFs. The magnified curves over the temperature range from 1100 to $1200{ }^{\circ} \mathrm{C}$ show the stable degree of degradability. First derivative plots for five silk samples were computed using Origin Pro 8 software.

\section{Transmission electron microscopy}

A small amount of degummed silk sample was digested using concentrated nitric acid into short fibers for the observation of the CNTs in the nanofibrils. The carbonized SFs were ground into a graphitic powder in an agate mortar and immersed in ethanol. Then, the dissolved SFs, graphitized solution and feeding additives were respectively dropped on a microgrid support membrane before observation using TEM. Nanoscale images were obtained to confirm the nanostructures of CNTs and the graphitization of SFs, and even LGS coated on CNTs, through transmission electron microscopy (JEOL JEM-2100, Japan) at $200 \mathrm{kV}$ with a series of matched magnifications from $25 \mathrm{k}-400 \mathrm{k}$.

\section{Raman spectroscopy}

Before measuring, dilute SF solution was dropped onto a silicon chip, and powder carbonized at a high-temperature was attached to the silicon chip with conductive glue. Subsequently, they were observed to confirm the presence of carbon, with spectral peaks at $\sim 1350$ and $\sim 1580 \mathrm{~cm}^{-1}$, using a Raman spectrometer (HORIBA Scientific Lab-RAM HR Evolution, Japan) with a laser wavelength of $532 \mathrm{~nm}$, over a scanning range from 200 to $3000 \mathrm{~cm}^{-1}$. All experiments were carried out at room temperature $\left(25^{\circ} \mathrm{C}\right)$.

\section{Fourier transform infrared spectroscopy}

The spectral peaks of SFs were recorded using a Fourier transform infrared spectrometer (FTIR Nicolet iS50, U.S.), which was equipped with a planar electromagnetic driven Michelson interferometer and attenuated total reflection (ATR) accessory. For each measurement, a sample was prepared by grinding $0.5 \%$ sample $\mathrm{w} / \mathrm{w}$ with dried $\mathrm{KBr}$ powder. FTIR spectroscopy was performed, scanning 64 scans with a resolution of $4 \mathrm{~cm}^{-1}$ over the wavenumber range of 400 to $4000 \mathrm{~cm}^{-1}$. This included measuring the IR spectrum of Amide I (1575-1725 $\mathrm{cm}^{-1}$ ), which was deconvoluted into three fitted Gaussian peaks at $\sim 1639 \mathrm{~cm}^{-1}$ for $\beta$-sheets, $\sim 1680 \mathrm{~cm}^{-1}$ for random coils or $\alpha$ helices, and $\sim 1700 \mathrm{~cm}^{-1}$ for $\beta$-turns.

\section{X-ray diffraction}

XRD patterns of clusters of aligned degummed SFs were obtained with an X-ray diffraction spectrometer (Bruker D8 Advance, Germany) using $\mathrm{Cu}-\mathrm{K} \alpha$ radiation $(\lambda=0.154 \mathrm{~nm})$. Each sample was independently scanned over a $2 \theta$ range from $10^{\circ}$ to $50^{\circ}$ with an interval of $0.02^{\circ}$ and a sample-to-detector distance of $60 \mathrm{~mm}$. The crystallographic indices, corresponding to baseline-corrected patterns of pristine and carbonized silk fibers, were determined from the radial intensities along the equator.

\section{X-ray photoelectron spectroscopy}

A small amount of carbonized SF powder was uniformly distributed on a test module to prepare a moderate film. XPS survey spectra were recorded via X-ray photoelectron spectroscopy (Thermo Fisher Scientific ESCALAB Xi+, U.S.) using radiation from a double anode $\mathrm{Al} / \mathrm{Mg}$ target with an interval of $0.05 \mathrm{eV}$ and a power of $400 \mathrm{~W}$. The high-resolution C1s spectra (282-293 eV) for each sample were simulated via AVANTAGE software to fit four Gaussian peaks for different bonds, including $\mathrm{C}-\mathrm{C}$ at $\sim 284.5 \mathrm{eV}, \mathrm{C}-\mathrm{N}$ at $\sim 285.3 \mathrm{eV}, \mathrm{C}-\mathrm{O}$ at $\sim 286.6 \mathrm{eV}$, and $\mathrm{C}=\mathrm{O}$ at $\sim 290 \mathrm{eV}$.

\section{Mechanical tests and electrical conductivity}

For each type of silk, 20 samples (two silk fibers randomly selected from each cocoon, a total of 10 cocoons for each group) were studied to obtain average values and standard deviations. The mechanical tensile strengths of degummed single SFs were separately measured using an INSTRON 5848 MicroTester (U.S.) with a $0.5 \mathrm{~N}$ load cell (force resolution corresponding to $0.5 \% \mathrm{~N}$, strain resolution corresponding to $0.02 \mu \mathrm{m}$ ) to obtain strainstress curves. In this work, a tensile rate of $3 \mathrm{~mm} \mathrm{~min}^{-1}$ and a gauge length of $20 \mathrm{~mm}$ were used. Measurements were all carried out at room temperature and a humidity of $50 \%$. Furthermore, SFs were heated in a tube furnace under an argon atmosphere (purity: 99.99\%; gas flow: $100 \mathrm{~cm}^{3} \mathrm{~min}^{-1}$ ) from room temperature to $350{ }^{\circ} \mathrm{C}$, and the temperature was held for 2.5 hours; then the temperature was increased from 350 to $900{ }^{\circ} \mathrm{C}$ and held for an additional 2 hours to form conjugated hexagonal rings of graphitization. Obtained carbonized silk fibers from each group were ground into graphitic powder in an agate mortar to measure conductivity using a digital multimeter.

\section{Results and discussion}

\section{Mechanical properties and structural behavior of degummed silk fibers}

We provide a scheme (Fig. 1A, top) showing the potential strategy for reinforcing silk fibers by feeding purified CNT additives to silkworms. The use of LGS as a surfactant to modify CNTs allows them to be dispersed in deionized water. In this work, we designed a feasible and facile purification method via multiple filtration through $0.2 \mu \mathrm{m}$ filters to improve the density of CNTs, which will largely increase the CNT content in silk fibers. The feeding of artificial additives included two groups fed SWCNTs with solution concentrations of $0.2 \mathrm{wt} \%$, with and without purification, and another two groups fed MWCNTs with solution concentrations of $0.2 \mathrm{wt} \%$, with and without purification. The resulting silk samples were respectively labeled SWCNT1-S, SWCNT2-S, MWCNT1-S and MWCNT2-S. An 
A)
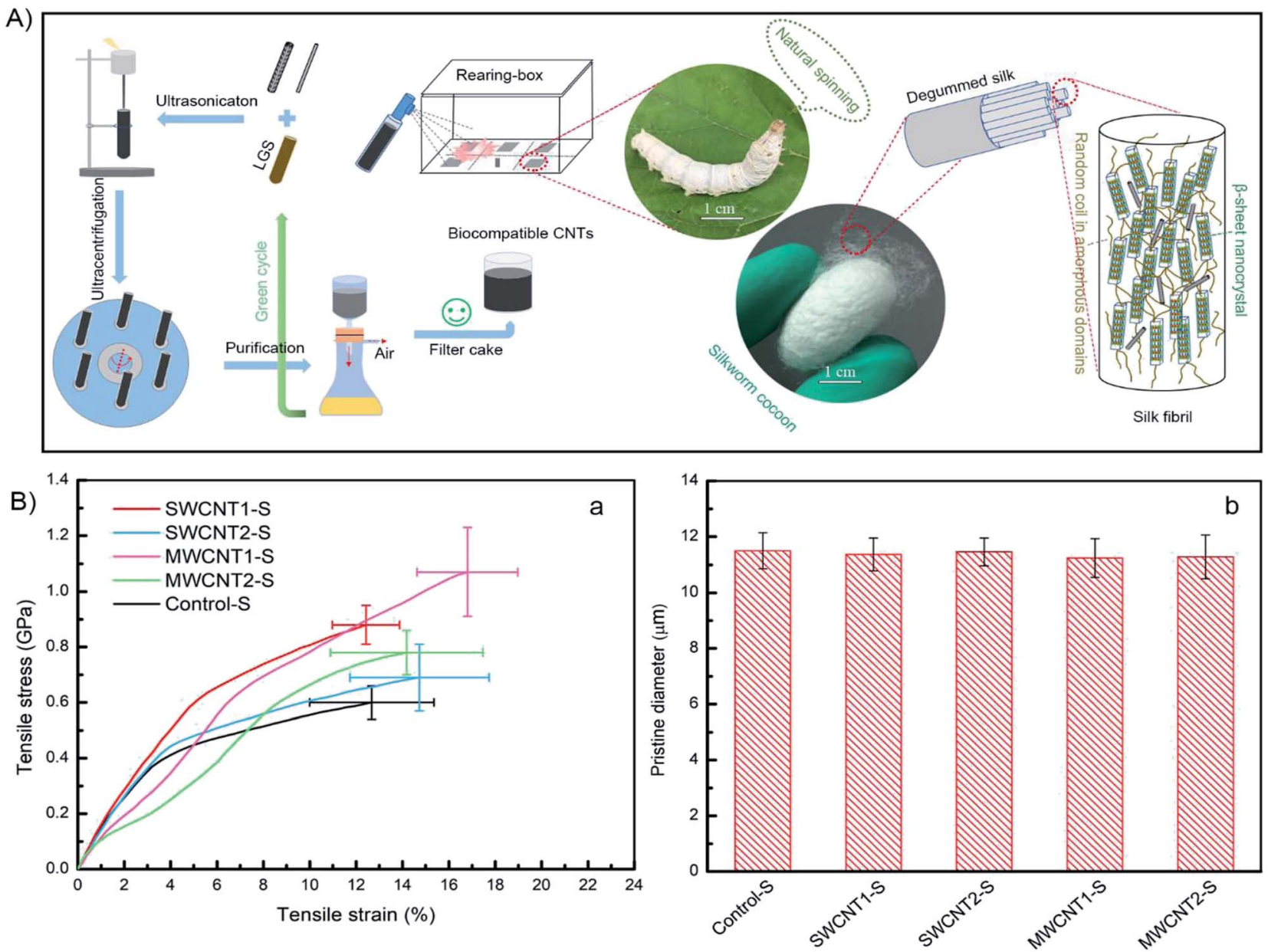

Fig. 1 Purification additives promoting the synthesis of high mechanical performance fibers through natural feeding. (A) The purification procedure for high density CNT solution using multiple filtration with $0.2 \mu \mathrm{m}$ filters. (B) (a) Mechanical curves from degummed silk fibers. The error bars denote standard deviations of stress at fracture (vertical axis) and strain at fracture (horizontal axis); (b) pristine diameters, showing the negligible influence of the incorporated CNT content on the silkworm spinning process.

additional additive involving pure LGS was provided as a control group. A total of $50 \mathrm{~B}$. mori silkworm larvae were randomly divided into five groups and naturally reared at the beginning of the first day of the fifth instar. After the spinning period, the cocoons were collected to remove the sericin coating and obtain degummed silk fibers. We studied the collected silk fibers in terms of specific areas, such as the feeding environment, tensile parameters and statistical methods, because different conditions can give rise to diverse as-obtained silk fibers. ${ }^{39,40}$

SWCNT1-S fibers exhibited a mechanical strength of $0.88 \mathrm{GPa}$ and strain of $12.43 \%$, but MWCNT1-S showed a mechanical strength as high as $1.07 \mathrm{GPa}$ with a strain of $16.80 \%$, indicating that the reinforced silk fibers benefited from the purified additives (Fig. 1B(a)). The toughness modulus of SWCNT1-S was enhanced to $6.83 \mathrm{GJ} \mathrm{m}^{-3}$, a 1.06-fold increase in comparison to SWCNT2-S. However, MWCNT1-S had a toughness modulus of $11.32 \mathrm{GJ} \mathrm{m}^{-3}$, which is an increase as high as 1.69-fold in comparison to MWCNT2-S, showing the large CNT content in the silk fibers due to purification. Thus, the incorporation of a large amount of CNTs influences the molecular kinetics and improves the mechanical properties. ${ }^{41}$ The stresses of SWCNT-S and MWCNT-S show different increases, from a minimum of $0.09 \mathrm{GPa}$ to a maximum of $0.47 \mathrm{GPa}$, in comparison to the Control-S group value of $0.60 \mathrm{GPa}$. More details are shown in Tables S4-S8 $\uparrow$ and Table 2, which show the mechanical properties of the obtained silk fibers.

In terms of the structural-property relationship at fracture, we found that the rupture shape, defined as the radial shape change, may explain the nanocrystalline mechanical behavior of the silk fibers. While protein structural systems swell upon hydration, tensile silk fibers will shrink along the force axis. ${ }^{42}$ Fig. $1 \mathrm{~B}(\mathrm{~b})$ and $2 \mathrm{C}$ show that there is no observable difference between the silk fiber diameters, suggesting that feeding additives of purified or unpurified nanofillers does not affect silkworm spinning process. Fig. $2 \mathrm{~A}$ illustrates that the molecular dynamics of a silk fibril, a unit filament of a silk fiber, may be subject to the influence of the CNT content, which can play a significant role as a source of frictional functionality. This includes the presence of random coils within amorphous domains and hydrogen bonds within nanocrystal domains hindering ruptures. ${ }^{1,43}$ As shown in Fig. $2 \mathrm{~B}$, the radial shapes of 
Table 2 Mechanical properties of degummed B. mori silk fibers

\begin{tabular}{lllr}
\hline Silk sample & Stress at fracture [GPa] & Strain at fracture [\%] & Toughness modulus [GPa] \\
\hline SWCNT1-S & 0.88 & 12.43 & 6.83 \\
SWCNT2-S & 0.69 & 14.73 & 6.40 \\
MWCNT1-S & 1.07 & 16.80 & 11.32 \\
MWCNT2-S & 0.78 & 14.18 & 6.67 \\
Control-S & 0.60 & 12.67 & 4.79
\end{tabular}

the cross-sectional ruptures, mostly flat or oval, can be ascribed to different CNT concentrations in silk fibers. In this work, purification contributes to a high CNT concentration, which internally prompts the reorganization of protein systems and subtly adjusts the molecular dynamics of supercontraction. ${ }^{44}$ It is evident that the molecular forces are heavily unaligned, ${ }^{45}$
A)

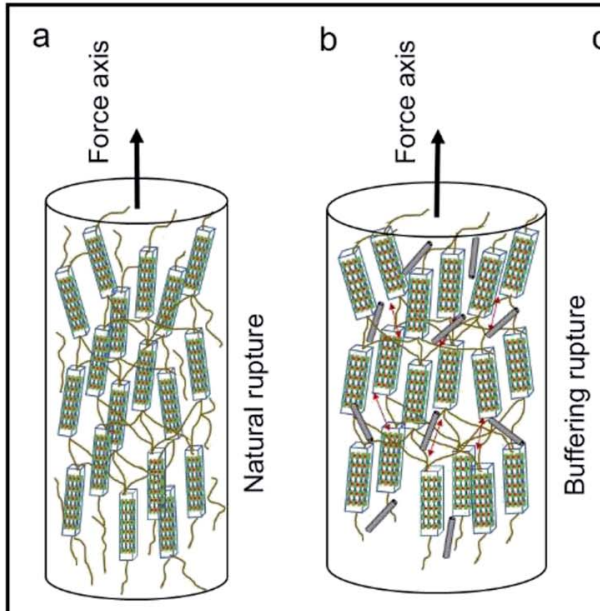

\section{C}
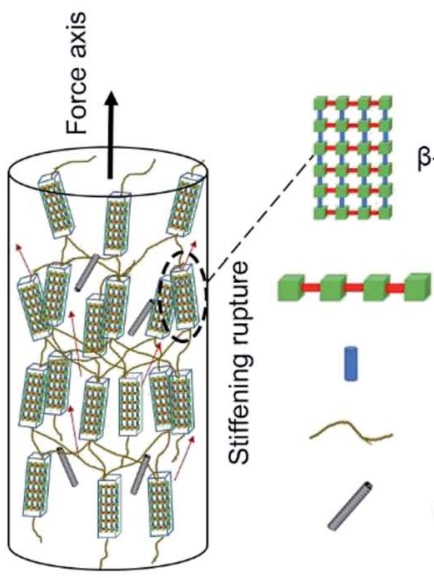

$\beta$-sheet nanocrystal

Peptide chain

Hydrogen bond

Random coil

Carbon nanotube
B)

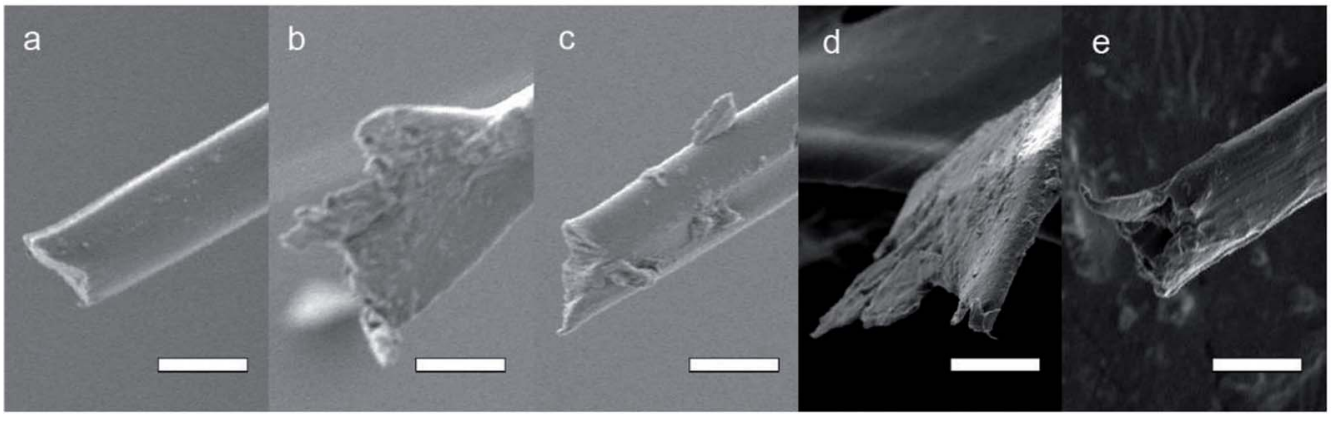

C)

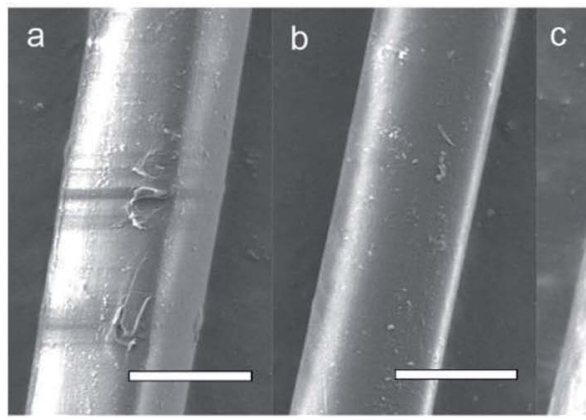

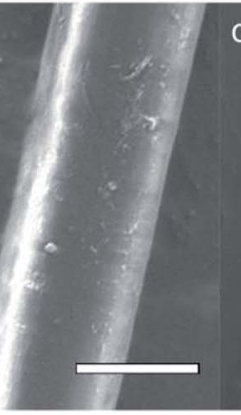

d

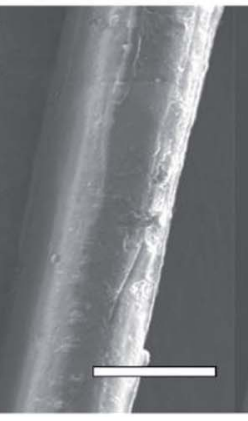

e

Fig. 2 Structural behavior mechanism and cross-sectional morphology with or without the rupturing of silk fibers. (A) (a) Silk fibrils without CNTs, called Control-S, under the force function; (b) silk fibrils with a high density of CNTs under the force function (the interactive forces in the amorphous domains are shown via bidirectional red arrows); (c) silk fibrils with a low density of CNTs under the force function (the internal forces in the amorphous domains are shown via unidirectional red arrows). (B) (a-e) Typical FESEM images of cross-sectional rupturing, giving a flat or oval morphology: the samples shown are Control-S, SWCNT1-S, SWCNT2-S, MWCNT1-S, and MWCNT2-S, respectively; scale bars: 10 Mm. (C) (a-e) FESEM images of pristine silk fibers: Control-S, SWCNT1-S, SWCNT2-S, MWCNT1-S and MWCNT2-S, respectively; scale bars: $10 \mu$ m. 
leading to different mechanical properties. The increased CNT content in the silk fiber samples SWCNT1-S and MWCNT1-S leads to the self-assembly of random coils or $\alpha$-helices into more movable knots $^{46}$ causing buffering-type ruptures (Fig. 2B(b and d)). On the other hand, the increasing orientation of molecular chains in the silk fibrils causes the protein reorganization to undergo close contraction, leading to a flat shape and the formation of buffering-type ruptures. On the contrary, SWCNT2-S and MWCNT2-S samples contain less CNTs in amorphous domains, resulting in an oval shape and leading to stiffening-type ruptures (Fig. 2B(c and e)). Besides, the Control-S sample, with no incorporated CNTs, shows more stiffening-type ruptures because there are no additional movable knots (Fig. 2B(a)).

\section{Thermal decomposition and the CNT content of degummed silk fibers}

The TGA curves in Fig. 3a show the degummed silk fibers over a temperature range from 25 to $1200{ }^{\circ} \mathrm{C}$, and the expansion given in the inset shows that the residue mass is almost steady from 1100 to $1200{ }^{\circ} \mathrm{C}$. We used a purification procedure to improve the CNT density in the artificial additives, but the CNT content in the silk fibers must still be confirmed. A silk fibroin consists of light- and heavy-chain polypeptides linkages and, from the first derivative curves in Fig. 3b, the major thermolysis of light chains and part of the heavy chains occurs in the temperature range between $230{ }^{\circ} \mathrm{C}$ and $550{ }^{\circ} \mathrm{C} .4^{47,48} \mathrm{We}$ also understand that the former stage between $25{ }^{\circ} \mathrm{C}$ and $230{ }^{\circ} \mathrm{C}$ involves the loss of water in semi-amorphous domains, and the latter stage of thermal decomposition is attributed to the few heavy-chain polypeptides and most of the incorporated CNTs, which are extremely hard to degrade. In detail, the thermal terminal curves from 1100 to $1200{ }^{\circ} \mathrm{C}$ shown in the expansion in Fig. 3a are steady, and their derivative plots are close to zero in Fig. 3b. Since at $1200{ }^{\circ} \mathrm{C}$ silk proteins are completely pyrolyzed, the other residue mass must be due to CNTs. Thus, in comparison to the decomposed Control-S, the residue mass of each decomposed sample showed an increased CNT content in
SWCNT1-S, SWCNT2-S, MWCNT1-S and MWCNT2-S. The SWCNT content in SWCNT1-S was considerably increased, 1.5fold, when compared to SWCNT2-S. Similarly, the MWCNT content in MWCNT1-S was 1.3-fold that in MWCNT2-S. As a result, the CNT content in silk fibers was largely increased through feeding with purified additives.

\section{Morphology and Raman spectra of the CNTs doped into degummed silk fibers}

TGA has affirmed the CNT content in the silk fibers, but it is essential to observe them directly. SWCNTs and MWCNTs in silk fibers were found using HRTEM, as shown in Fig. 4a and b. Their diameters are estimated to be $\sim 1.9 \mathrm{~nm}$ and $\sim 16 \mathrm{~nm}$, respectively, which is in good agreement with the diameters of the purchased CNTs. We also found that the SWCNTs and MWCNTs were embedded in the silk fibroins, and kept their pristine forms, indicating that both types of CNT interfered with the localized environment during silk formation. Raman spectra curves of silk fibroins, excrement, and pristine CNTs were obtained to confirm the spectral peaks from the CNTs. Fig. 4c verifies the existence of SWCNTs. The radial breathing mode peak at $\sim 267 \mathrm{~cm}^{-1}$, D-band at $\sim 1346 \mathrm{~cm}^{-1}$, G-band at $\sim 1588 \mathrm{~cm}^{-1}$, and $\mathrm{G}^{\prime}$-band at $\sim 2663 \mathrm{~cm}^{-1}$ (ref. 30, 49 and 50) indicate that the SWCNTs are really incorporated into the silk fibers, while some CNTs were also present in the excrement. Similarly, in Fig. 4d, the D-band at $\sim 1349 \mathrm{~cm}^{-1}$, G-band at $\sim 1589 \mathrm{~cm}^{-1}$ and $\mathrm{G}^{\prime}$-band at $\sim 2699 \mathrm{~cm}^{-1}$ indicate that MWCNTs are also embedded in the silk fibers. HRTEM images combined with Raman analysis demonstrate that CNTs can be embedded into silk fibers through natural rearing with artificial additives.

\section{Fibroin structural evolution of degummed silk fibers}

Natural silk fibers exhibit ordinary strength and toughness, as a result of their innate secondary structures composed of $\beta$ sheets, random coils or $\alpha$-helices, and $\beta$-turns. Reorganization among these three elements establishes a movable molecular network, ${ }^{\mathbf{4 1}, 51}$ leading to reinforced toughness. Although random coils and $\alpha$-helices help crosslinking, more $\beta$-sheets or
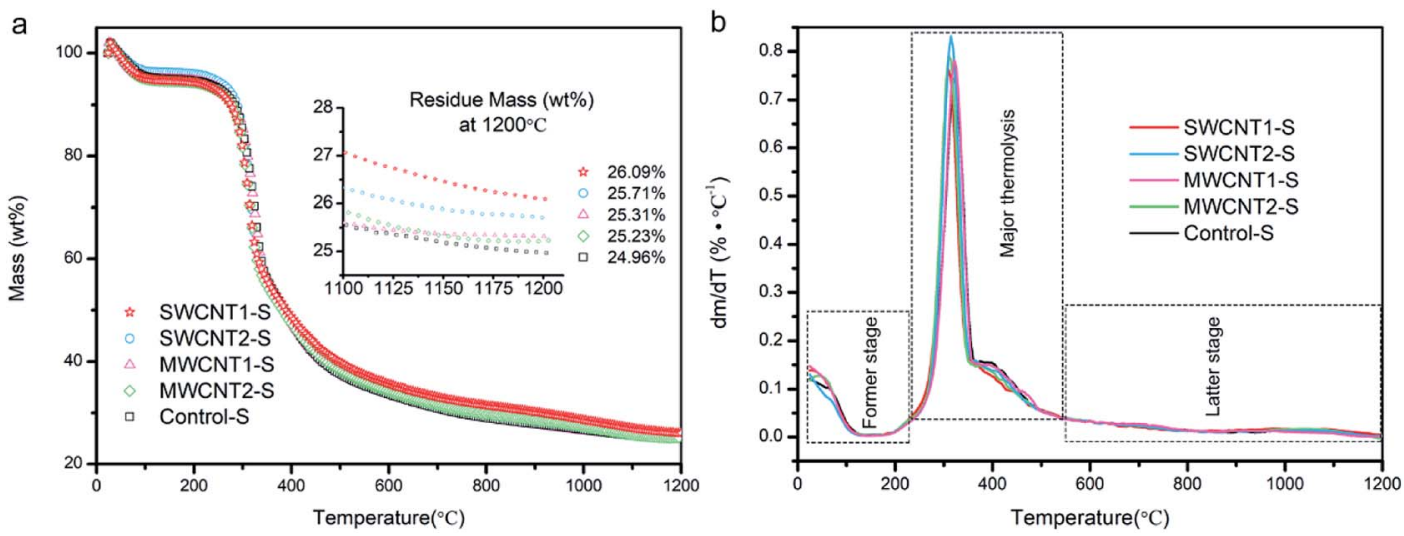

Fig. 3 The influence of high temperature on the thermal stability of SWCNT-S and MWCNT-S. (a) Thermogravimetric scans of the silk fibers showing major decomposition between $230^{\circ} \mathrm{C}$ and $550^{\circ} \mathrm{C}$. The inset shows expanded curves from (a) over a temperature range from 1100 to $1200^{\circ} \mathrm{C}$. (b) First derivative plots of the TGA curves of five silk samples. 
a

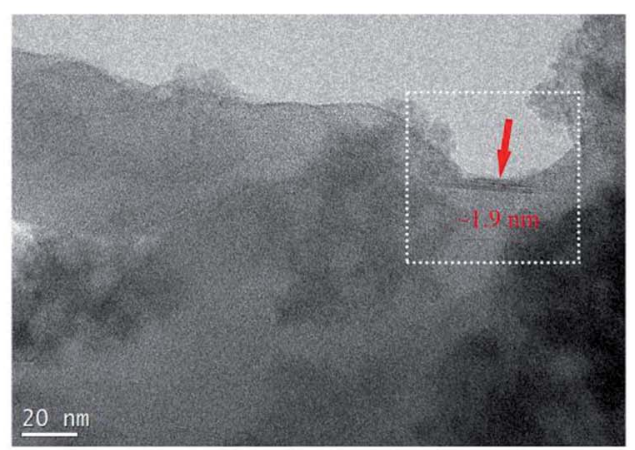

c

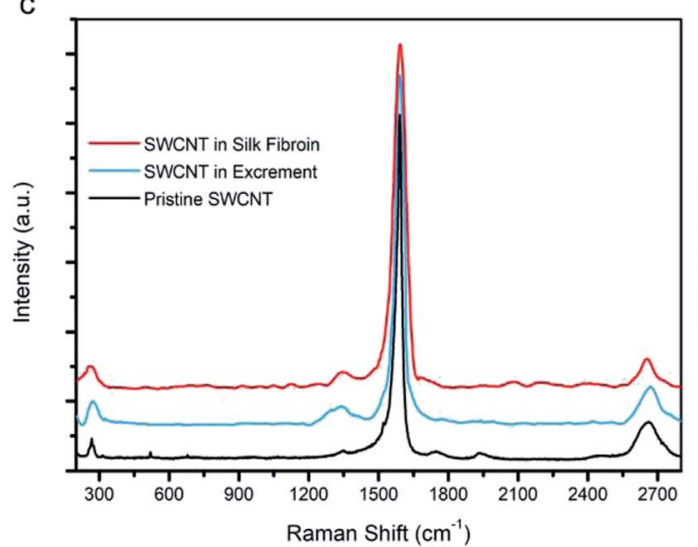

b

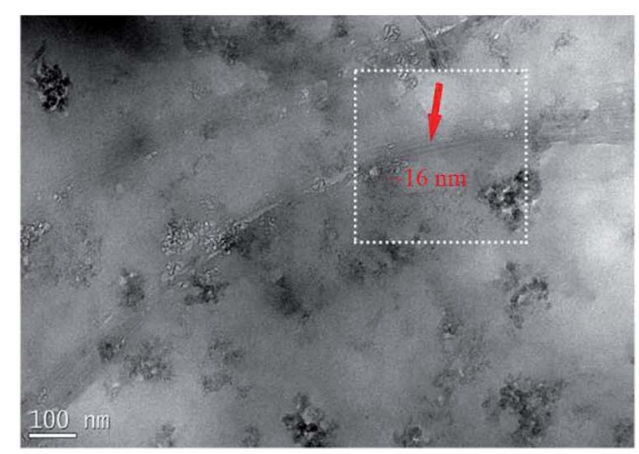

d

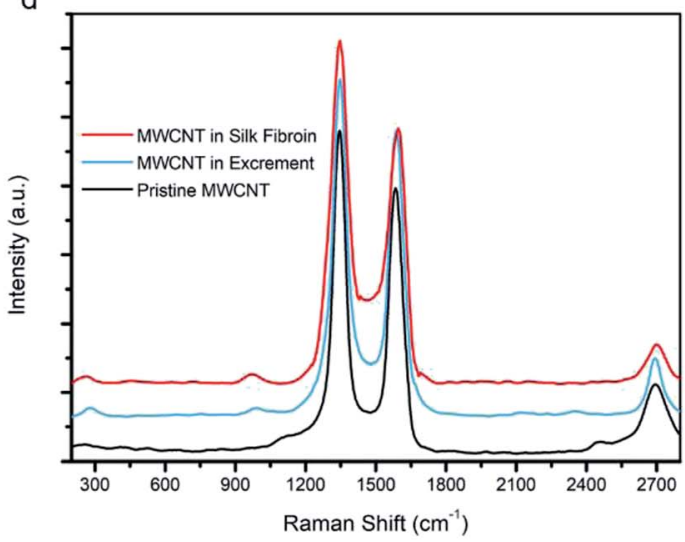

Fig. 4 Transmission electron microscopy (TEM) images of CNTs in silk fibroins and Raman spectroscopy of CNTs in silk fibroins and excrement in comparison to pristine CNTs. (a) A TEM image of SWCNTs in SWCNT-S. (b) A TEM image of MWCNTs in MWCNT-S. (c) Raman spectra curves of silk fibroin and excrement obtained after feeding with the SWCNT additive, and pristine SWCNTs. (d) Raman spectra curves of silk fibroin and excrement obtained after feeding with the MWCNT additive, and pristine MWCNTs.

embedded CNTs contribute to the formation of "trusty knots". When more CNTs are embedded in the silk fibers, the hierarchical evolution gives rise to more $\beta$-sheet structures and the formation of more trusty knots in the silk matrix. The $\beta$-sheets are the fold pleats, while the $\alpha$-helices act as cords to crosslink with CNTs and create novel frictional knots. Fourier transform infrared spectroscopy (FTIR) helps to elucidate the subtle evolution of the secondary structures. The spectra in Fig. 5a of five silk samples show a slight shifting of the center peaks. The FTIR spectrum of the amide I region, from 1575 to $1725 \mathrm{~cm}^{-1}$, including the peaks at $1639 \mathrm{~cm}^{-1}$ for $\beta$-sheets, $1680 \mathrm{~cm}^{-1}$ for random coils or $\alpha$-helices, and $1700 \mathrm{~cm}^{-1}$ for $\beta$-turns, helps elucidate secondary structures. ${ }^{52,53}$

Fig. 5b shows the content of each type of secondary structure in the deconvoluted spectra. The increased proportion of $\beta$ sheets in SWCNT1-S and MWCNT1-S indicates the secondary transformation to $\beta$-sheet nanocrystals within the amorphous domains. Moreover, the proportion of $\beta$-turns in the amorphous domains correspondingly tends to be lower in SWCNT1-S and MWCNT1-S as a result of the high CNT content. It has been reported that noncovalent interactions between CNTs and zwitterionic amino acids can result in structural molecular forces,$^{54}$ including van der Waals forces, hydrogen bonds, and $\pi-\pi^{*}$ stacking. Due to molecular interactions arising from the CNTs, high-efficiency knots formed via self-assembly from random coils or $\alpha$-helices leads to additional CNT-knots. Thus, an increase in CNTs and $\beta$-sheets can synergistically create more trusty knots to hinder macromolecular chain ruptures. In this regard, we highlight that CNTs are effective in adjusting molecule structures, thus leading to better mechanical performance. Detailed information is shown in Fig. S3 and Table S1. $\dagger$

\section{XRD patterns of degummed silk fibers at $25{ }^{\circ} \mathrm{C}$ and $900{ }^{\circ} \mathrm{C}$}

Due to their application in smart flexible sensors for human health, carbonized fibers from green biological materials have drawn prevalent attention. ${ }^{13,55}$ Fig. $6 \mathrm{a}$ and b show XRD patterns from non-carbonized silk samples at $25{ }^{\circ} \mathrm{C}$ and carbonized silk samples at $900{ }^{\circ} \mathrm{C}$ using transformative indices of crystallographic planes. According to previous reports, the main distribution of intensity in such XRD patterns includes the halo produced by amorphous domains and the Bragg peaks produced by $\beta$-sheet nanocrystals. ${ }^{56} \mathrm{Fig}$. 6 a shows XRD patterns of non-carbonized silk samples, with characteristic peaks from the main crystallographic planes of (020) and (210), in comparison to the (110) reflection. ${ }^{57,58}$ The spectra indicate that the embedded CNTs in the silk fibers contribute to the major formation of $\beta$-sheet nanocrystals. Moreover, the (211) plane family shows little observable difference due to the weak orientation of unaggregated $\beta$-sheets. In contrast, Fig. $6 \mathrm{~b}$ shows the transformation of the carbonized silk protein toward the (002) plane of graphitic carbon, in agreement with reports that 

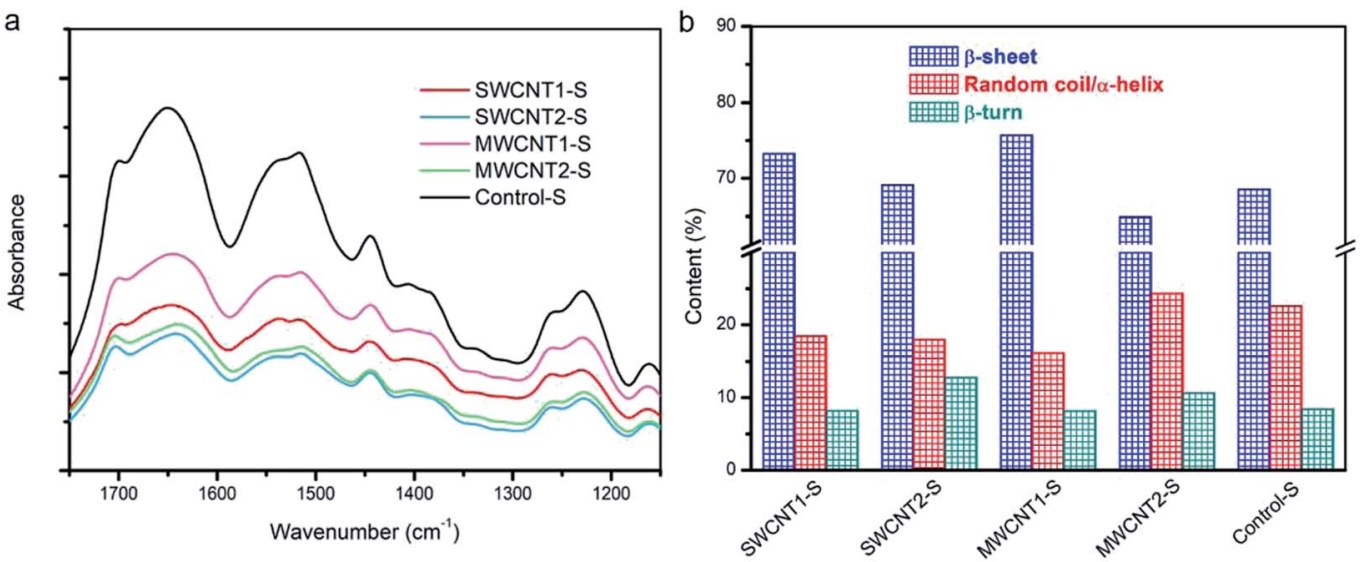

Fig. 5 Influence of the incorporated SWCNTs or MWCNTs on the secondary structures of the silk fibers. (a) FTIR curves showing the different absorbances of SWCNT1-S, SWCNT2-S, MWCNT1-S, MWCNT2-S, and Control-S. (b) The secondary structure content values for five silk samples obtained from amide I spectra.

high temperature induces the formation of conjugated $\mathrm{sp}^{2}-$ hybridized carbon hexagonal rings. ${ }^{59,60}$ It has been established that the (002) plane of carbonized silk protein should exhibit a sharp peak under ultrahigh temperature conditions. However, at $900{ }^{\circ} \mathrm{C}$ the graphitic stacking of the (002) plane at around $25.82^{\circ}$ is not observed, indicating larger carbonaceous interplanar distances. ${ }^{61}$ Hence, the carbonized structures should be studied further to provide a rational explanation.
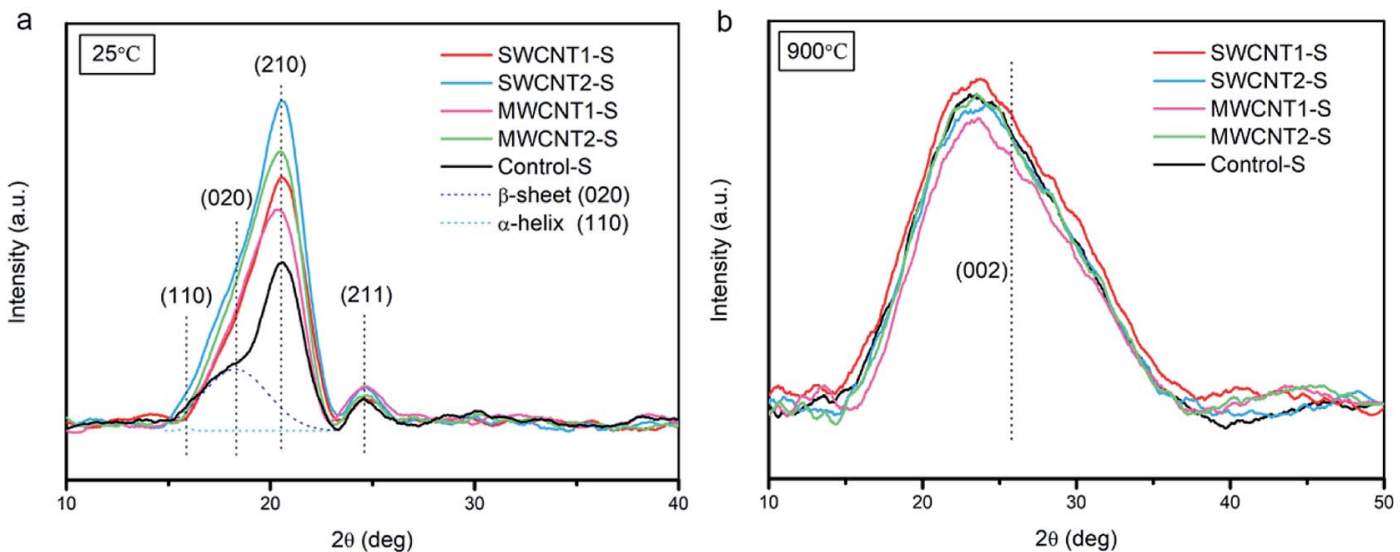

c

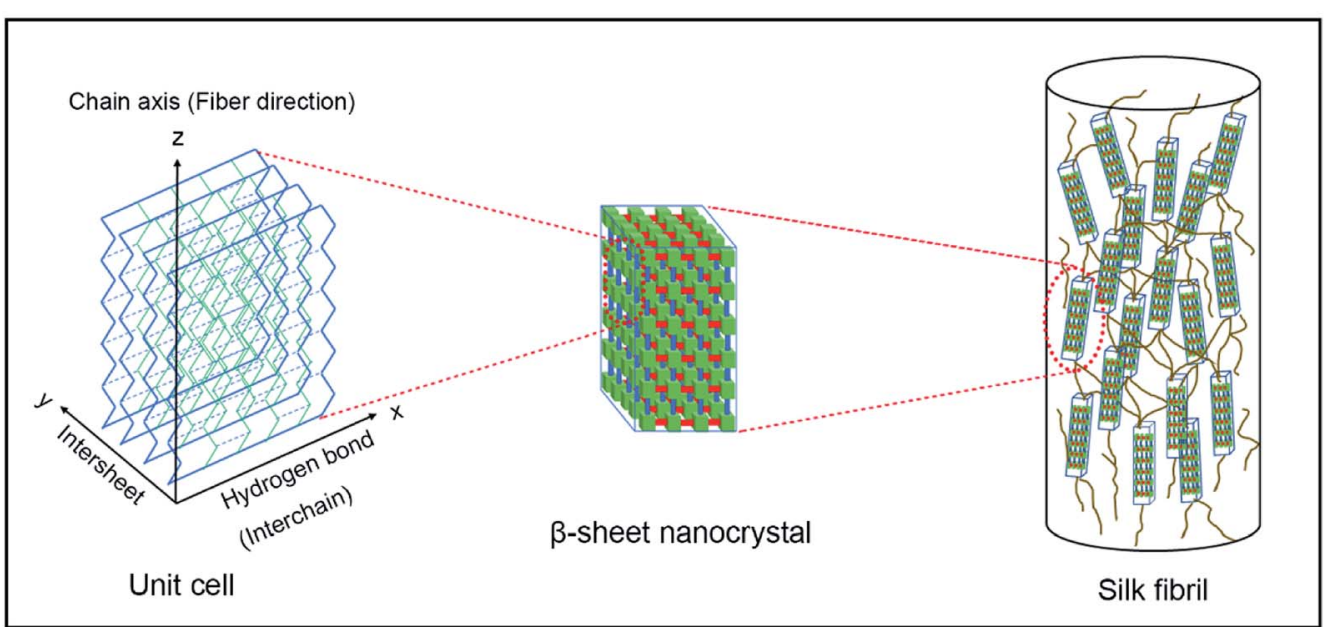

Fig. 6 XRD patterns of silk fibers at different temperatures. (a) XRD spectra of silk fibers at $25^{\circ} \mathrm{C}$, showing their original condition. (b) XRD spectra of silk fibers heated at $900^{\circ} \mathrm{C}$, showing their carbonized condition. (c) A sketch showing the unit cell of a $\beta$-sheet nanocrystal (xyz) along the silk fibril axis. 


\section{Conductivity and characterization of the graphitized degummed silk fibers}

To get a better understanding of the electrical conductivity arising from the graphitized structures, we investigated the Raman spectra of carbonized silk fibers, as shown in Fig. 7A(a and $\mathrm{b})$. The $I_{\mathrm{G}} / I_{\mathrm{D}}$ ratio between the G-band of graphitized carbon at $\sim 1580 \mathrm{~cm}^{-1}$ and the D-band of disordered carbon at $\sim 1350 \mathrm{~cm}^{-1}$ is widely used to quantify the degree of nanoscale graphitization. ${ }^{61,62}$ Here, as shown in the left inset graph, the $I_{\mathrm{G}} /$ $I_{\mathrm{D}}$ ratio of SWCNT1-S is $\sim 1.046$, slightly larger than that of SWCNT2-S ( 1.037). Similarly, as can be seen in the right inset graph, the $I_{\mathrm{G}} / I_{\mathrm{D}}$ ratio of MWCNT1-S is $\sim 1.004$, also slightly larger than that of MWCNT2-S ( 0.993). The enhancement in the $I_{\mathrm{G}} / I_{\mathrm{D}}$ ratio suggests an increase in the graphitization degree, resulting from the larger CNT content in the silk fibers. Moreover, Control-S shows the lowest $I_{\mathrm{G}} / I_{\mathrm{D}}$ ratio $(\sim 0.967)$, indicating that more defects are induced during carbonization due to the absence of incorporated CNTs in the silk fibers. It is evident that the purification process, which removes most of the excess LGS, enhances the CNT content in the silk fibers and leads to an increase in the graphitization degree.

According to high-resolution TEM images of carbonized silk samples, the incorporated CNTs influence the density of $\mathrm{sp}^{2}$ hybridized hexagonal graphite, because SWCNT1-S had larger interplanar spacing of $\sim 0.39 \mathrm{~nm}$, while MWCNT1-S exhibited smaller interplanar spacing of $\sim 0.32 \mathrm{~nm}$ (Fig. 7B(a and c)). ${ }^{13,59}$ We quantitatively analyzed the element content values using the high resolution $\mathrm{C}$ 1s XPS line, and found that Control-S has the highest $\mathrm{C}-\mathrm{C}$ content (Fig. 7C(a)), which is likely to arise from disordered carbonaceous residue. On the contrary, the decrease in the $\mathrm{C}-\mathrm{C}$ content in SWCNT1-S and MWCNT1-S revealed the larger CNT content in the silk fibers, which leads to the formation of an ordered graphitized structure. The detailed
A)

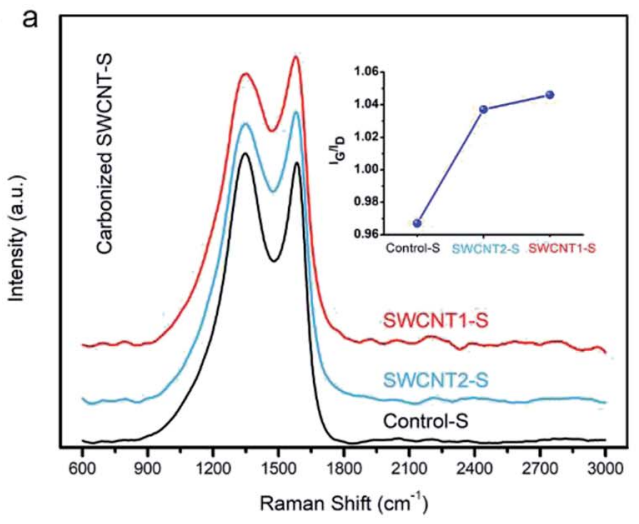

$\mathrm{b}$

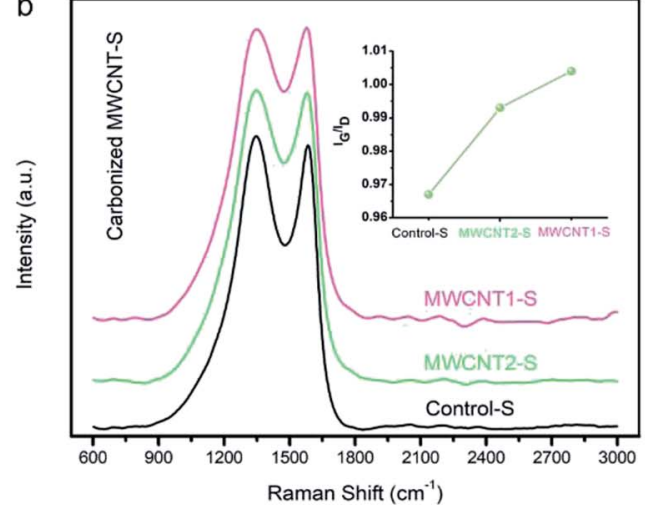

B)

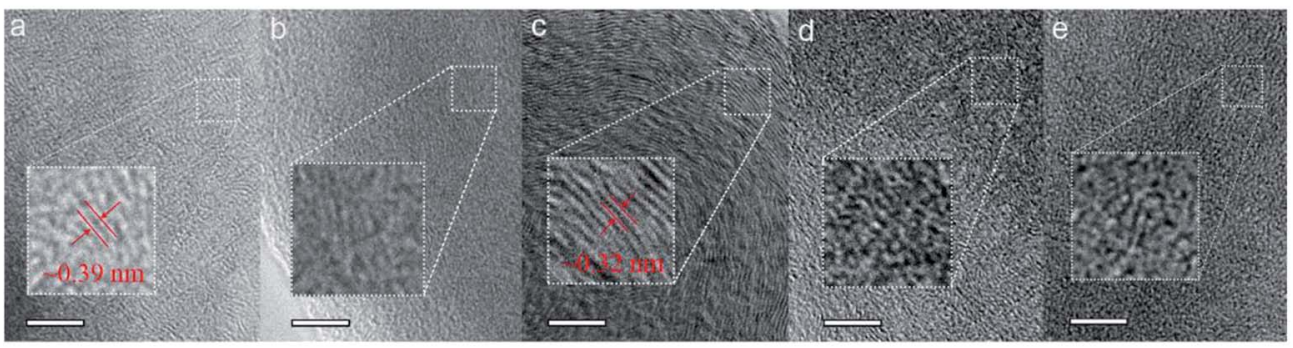

C)

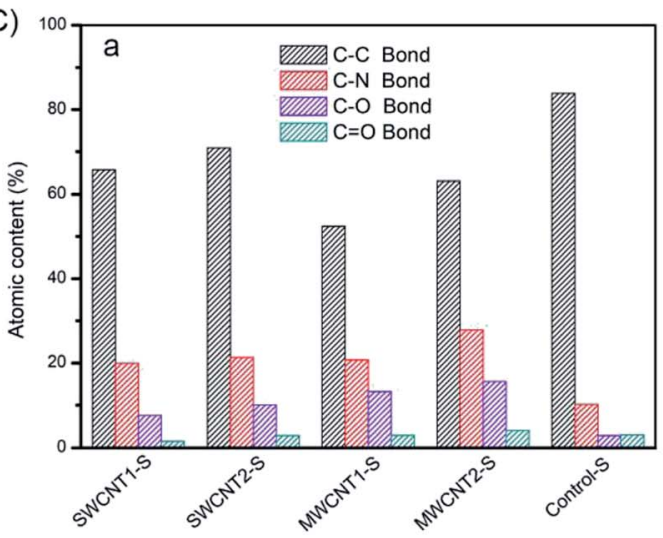

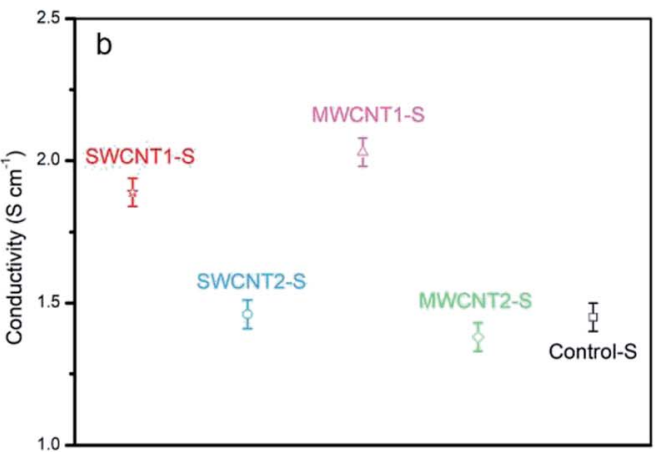

Fig. 7 The conductivity and characterization of graphitized silk. (A) (a and b) Raman curves from carbonized silk with and without purification. The inset graphs show the G-band to D-band $\left(I_{G} / I_{D}\right)$ intensity ratio. (B) (a-e) High-resolution TEM images of graphitized nanostructures from SWCNT1-S, SWCNT2-S, MWCNT1-S, MWCNT2-S and Control-S; scale bars: $5 \mathrm{~nm}$. (C) (a) The deconvoluted results of the atomic content values based on the $C$ 1s spectrum from XPS; (b) electrical conductivity results, showing the influence of graphitization with and without purification. 
statistics are shown in Fig. S4 and Table S2. $\dagger$ We also measured the conductivities of carbonized SWCNT1-S $\left(1.89 \mathrm{~S} \mathrm{~cm}^{-1}\right)$ and carbonized MWCNT1-S (2.03 $\left.\mathrm{S} \mathrm{cm}^{-1}\right)$, as shown in Fig. $7 \mathrm{C}(\mathrm{b})$; these values mostly originate from the abundant secondary structures. It is noteworthy that Control-S shows a conductivity of $1.45 \mathrm{~S} \mathrm{~cm} \mathrm{~cm}^{-1}$, indicating that silk fibers have intrinsic conductivity in the absence of CNTs. Detailed data are shown in Fig. S5, $\uparrow$ and the statistics are exhibited in Table S9. $\dagger$ With this understanding of the relationship between conductivity and graphitization, this valid and facile strategy to improve the CNT content of natural silk fibers can be further explored and applied to biocompatible sensors. ${ }^{63,64}$

\section{Conclusions}

As a remarkable biomaterial with a smooth texture, ecofriendly degradability and superior mechanical properties, silkworm fibers have been reinforced via feeding a CNT/LGS composite to B. mori silkworms. Gaining insight into previous studies with no focus on a purification strategy for artificial additives, in the present study we found that traditional feeding additives contain too much lignosulfonate (LGS) coating; excess LGS will block CNTs from entering into silk fibers. It is therefore essential to remove excess LGS. A comparative study with and without purification was carried out to clarify the effects of the incorporated CNTs on silk fibers. Purification led to a large increase in the CNT content and thus an increase in $\beta$-sheet nanocrystals as well. In addition, an increased amount of CNTs can self-assemble into additional frictional knots, providing a better understanding of the buffering behavior at fracture. We also demonstrated that the embedded CNTs led to an ordered graphitized structure within the silk fibers, contributing to conductivity enhancement. The mechanical performance and conductivity of MWCNT1-S are higher than those of SWCNT1-S, suggesting that MWCNTs are preferable for high performance fibers.

\section{Conflicts of interest}

There are no conflicts to declare.

\section{Acknowledgements}

This research was financially supported by the National Natural Science Foundation of China (No. 61275179 and 60970815), the Fundamental Research Funds for the Central Universities (No. xjj2015112), and the Natural Science Basic Research Plan in Shaanxi Province of China (Program No. 2016JM8047). We thank Dr Huimin Li from the School of Aerospace of Xi'an Jiaotong University and Engineer Yu Wang from the Instrument Analysis Center of Xi'an Jiaotong University for their assistance with mechanical tensile and Raman analysis, respectively.

\section{Notes and references}

1 L. Koh, Y. Cheng, C. Teng, Y. Khin, X. Loh, S. Tee, et al., Structures, mechanical properties and applications of silk fibroin materials, Prog. Polym. Sci., 2015, 46, 86-110.
2 C. Vepari and D. L. Kaplan, Silk as a biomaterial, Prog. Polym. Sci., 2007, 8-9(32), 991-1007.

3 D. Porter and F. Vollrath, Silk as a Biomimetic Ideal for Structural Polymers, Adv. Mater., 2009, 21, 487-492.

$4 \mathrm{~F}$. Vollrath and D. Porter, Silks as ancient models for modern polymers, Polymer, 2009, 24(50), 5623-5632.

5 S. Ghosh, S. T. Parker, X. Wang, D. L. Kaplan and J. A. Lewis, Direct-Write Assembly of Microperiodic Silk Fibroin Scaffolds for Tissue Engineering Applications, Adv. Funct. Mater., 2008, 18, 1883-1889.

6 G. H. Altman, R. L. Horan, H. H. Lu, J. Moreau and I. Martin Silk, Matrix for Tissue Engineered Anterior Cruciate Ligaments, Biomaterials, 2002, 23, 4131-4141.

7 K. Numata and D. L. Kaplan, Silk-based delivery systems of bioactive molecules, Adv. Drug Delivery Rev., 2010, 15(62), 1497-1508.

8 K. Tsioris, W. K. Raja, E. M. Pritchard, B. Panilaitis, D. L. Kaplan and F. G. Omenetto, Fabrication of Silk Microneedles for Controlled-Release Drug Delivery, Adv. Funct. Mater., 2012, 2(22), 330-335.

9 G. H. Altman, F. Diaz, C. Jakuba, T. Calabro, R. L. Horan, J. Chen, et al., Silk-based biomaterials, Biomaterials, 2003, 3(24), 401-416.

10 Z. Ding, H. Han, Z. Fan, H. Lu and Y. Sang, Nanoscale SilkHydroxyapatite Hydrogels for Injectable Bone Biomaterials, ACS Appl. Mater. Interfaces, 2017, 9, 16913-16921.

11 H. Tao, D. L. Kaplan and F. G. Omenetto Silk, Materials - A Road to Sustainable High Technology, Adv. Mater., 2012, 21(24), 2824-2837.

12 M. Hong, G. Choi, J. Kim, J. Jang, B. Choi, J. Kim, et al., Biomimetic Chitin-Silk Hybrids: An Optically Transparent Structural Platform for Wearable Devices and Advanced Electronics, Adv. Funct. Mater., 2017, 1705480.

13 C. Wang, X. Li, E. Gao, M. Jian, K. Xia, Q. Wang, et al., Carbonized Silk Fabric for Ultrastretchable, Highly Sensitive, and Wearable Strain Sensors, Adv. Mater., 2016, 31(28), 6640-6648.

14 C. Müller, M. Hamedi, R. Karlsson, R. Jansson and R. Marcilla Woven, Electrochemical Transistors on Silk Fibers, Adv. Mater., 2011, 23, 898-901.

15 Q. Wang, C. Wang, M. Zhang, M. Jian and Y. Zhang, Feeding Single-Walled Carbon Nanotubes or Graphene to Silkworms for Reinforced Silk Fibers, Nano Lett., 2016, 16, 6695-6700.

16 E. Lepore, F. Bosia, F. Bonaccorso, M. Bruna and S. Taioli, Spider silk reinforced by graphene or carbon nanotubes, 2D Mater, 2017, (4), 31013.

17 J. T. Wang, L. L. Li, M. Y. Zhang, S. L. Liu and L. H. Jiang, Directly obtaining high strength silk fiber from silkworm by feeding carbon nanotubes, Mater. Sci. Eng., C, 2014, 34, 417-421.

18 N. C. Tansil, Y. Li, C. P. Teng, S. Zhang and K. Y. Win, Intrinsically Colored and Luminescent Silk, Adv. Mater., 2011, 23, 1463-1466.

19 N. C. Tansil, L. D. Koh and M. Han, Functional Silk: Colored and Luminescent, Adv. Mater., 2012, 11(24), 1388-1397.

20 X. Wang, P. Zhao, Y. Li, Q. Yi, S. Ma, K. Xie, et al., Modifying the Mechanical Properties of Silk Fiber by Genetically 
Disrupting the Ionic Environment for Silk Formation, Biomacromolecules, 2015, 10(16), 3119-3125.

21 T. Iizuka, H. Sezutsu, K. Tatematsu, I. Kobayashi, N. Yonemura, K. Uchino, et al., Colored Fluorescent Silk Made by Transgenic Silkworms, Adv. Funct. Mater., 2013, 42(23), 5232-5239.

22 V. Nguyen, J. Si, L. Yan and X. Hou, Direct demonstration of photoluminescence originated from surface functional groups in carbon nanodots, Carbon, 2016, 108, 268-273.

23 B. Chen, P. Zhang, L. Ding, J. Han, S. Qiu, Q. Li, et al., Highly Uniform Carbon Nanotube Field-Effect Transistors and Medium Scale Integrated Circuits, Nano Lett., 2016, 8(16), 5120-5128.

24 J. Gu, J. Han, D. Liu, X. Yu, L. Kang, S. Qiu, et al., SolutionProcessable High-Purity Semiconducting SWCNTs for Large-Area Fabrication of High-Performance Thin-Film Transistors, Small, 2016, 36(12), 4993-4999.

25 X. Yao, G. Guo, P. Z. Li, Z. Z. Luo and Q. Yan, Scalable Synthesis of Honeycomblike $\mathrm{V}_{2} \mathrm{O}_{5}$ /Carbon Nanotube Networks as Enhanced Cathodes for Lithium-Ion Batteries, ACS Appl. Mater. Interfaces, 2017, 9, 42438-42443.

26 D. Shan, S. Deng, C. He, J. Li and H. Wang, Intercalation of rigid molecules between carbon nanotubes for adsorption enhancement of typical pharmaceuticals, Chem. Eng. J., 2018, 332, 102-108.

27 D. Zou, X. Ma, X. Liu, P. Zheng and Y. Hu, Thermal performance enhancement of composite phase change materials (PCM) using graphene and carbon nanotubes as additives for the potential application in lithium-ion power battery, Int. J. Heat Mass Transfer, 2018, 120, 33-41.

28 S. Iijima, Carbon nanotubes: past, present, and future, Phys. $B, 2002,1(323), 1-5$.

29 S. Iijima, C. Brabec, A. Maiti and J. Bernholc, Structural flexibility of carbon nanotubes, J. Chem. Phys., 1996, 5(104), 2089-2092.

30 T. Belin and F. Epron, Characterization methods of carbon nanotubes: a review, Mater. Sci. Eng., B, 2005, 2(119), 105118.

31 C. Zhang, Y. Zhang, H. Shao and X. Hu, Hybrid Silk Fibers Dry-Spun from Regenerated Silk Fibroin/Graphene Oxide Aqueous Solutions, ACS Appl. Mater. Interfaces, 2016, 8, 3349-3358.

32 G. Fang, Z. Zheng, J. Yao, M. Chen, Y. Tang, J. Zhong, et al., Tough protein-carbon nanotube hybrid fibers comparable to natural spider silks, J. Mater. Chem. B, 2015, 3, 3940-3947.

33 M. Kang, P. Chen and H. Jin, Preparation of multiwalled carbon nanotubes incorporated silk fibroin nanofibers by electrospinning, Curr. Appl. Phys., 2009, 1(9), S95-S97.

34 A. Rising, H. Nimmervoll, S. Grip, A. Fernandezarias and E. Storckenfeldt, Spider Silk Proteins - Mechanical Property and Gene Sequence, Zool. Sci., 2005, 22, 273-281.

35 R. Fedi, M. Urovec and F. Sehnal, Correlation between Fibroin Amino Acid Sequence and Physical Silk Properties, J. Biol. Chem., 2003, 278, 35255-35264.

36 J. M. Gosline, P. A. Guerette, C. S. Ortlepp and K. N. Savage, The mechanical design of spider silks: from fibroin sequence to mechanical function, J. Exp. Biol., 1999, 202(Pt 23), 3295-3303.

37 D. Tasis, N. Tagmatarchis, A. Bianco and M. Prato, Chemistry of Carbon Nanotubes, Chem. Rev., 2006, 3(106), 1105-1136.

38 X. Ouyang, X. Qiu and P. Chen, Physicochemical characterization of calcium lignosulfonate-a potentially useful water reducer, Colloids Surf., A, 2006, (282-283), 489-497.

39 Z. Shao and F. Vollrath, Surprising strength of silkworm silk, Nature, 2002, 418, 741.

40 F. Vollrath, B. Madsen and Z. Shao, The effect of spinning conditions on the mechanics of a spider's dragline silk, Proc. R. Soc. London, Ser. B, 2001, (268), 2339-2346.

41 C. Guo, J. Zhang, X. Wang, A. T. Nguyen, X. Y. Liu and D. L. Kaplan, Comparative Study of Strain-Dependent Structural Changes of Silkworm Silks: Insight into the Structural Origin of Strain-Stiffening, Small, 2017, 47(13), 1702266.

42 R. W. Work, A Comparative Study of the Super-Contraction of Major Ampullate Silk Fibers of Orb-Web-Building Spiders (Araneae), J. Arachnol., 1981, (9), 299-308.

43 N. Du, Z. Yang, X. Y. Liu, Y. Li and H. Y. Xu, Structural Origin of the Strain-Hardening of Spider Silk, Adv. Funct. Mater., 2011, 4(21), 772-778.

44 T. Giesa, R. Schuetz, P. Fratzl, M. J. Buehler and A. Masic, Unraveling the Molecular Requirements for Macroscopic Silk Supercontraction, ACS Nano, 2017, 11, 9750-9758.

45 I. Su and M. J. Buehler, Spider Silk Dynamic mechanics, Nat. Mater., 2016, 15, 1054.

46 A. Nova, S. Keten, N. M. Pugno, A. Redaelli and M. J. Buehler, Molecular and Nanostructural Mechanisms of Deformation, Strength and Toughness of Spider Silk Fibrils, Nano Lett., 2010, 7(10), 2626-2634.

47 S. Osaki, Thermal Properties of Spider's Thread, Acta Arachnol., 1989, (37), 69-75.

48 P. M. Cunniff, S. A. Fossey, M. A. Auerbach, J. W. Song, D. L. Kaplan, W. W. Adams, et al., Mechanical and Thermal Properties of Dragline Silk from the Spider, Polym. Adv. Technol., 1994, 5, 401-410.

49 Y. Zhang, H. Son, J. Zhang, M. S. Dresselhaus and J. Kong, Raman Spectra Variation of Partially Suspended Individual Single-Walled Carbon Nanotubes, J. Phys. Chem. C, 2007, 111, 1983-1987.

50 R. Saito, M. Hofmann, G. Dresselhaus, A. Jorio and M. S. Dresselhaus, Raman spectroscopy of graphene and carbon nanotubes, Adv. Phys., 2011, 3(60), 413-550.

51 A. Glišović, T. Vehoff, R. J. Davies and T. Salditt, Strain Dependent Structural Changes of Spider Dragline Silk, Macromolecules, 2008, 2(41), 390-398.

52 J. L. Cenis, R. Madurga, S. D. Aznar-Cervantes, A. A. LozanoPérez and N. Marí-Buyé, Mechanical behaviour and formation process of silkworm silk gut, Soft Matter, 2015, 11, 8981-8991.

53 S. Ling, Z. Qi, D. P. Knight, Z. Shao and X. Chen, Synchrotron FTIR microspectroscopy of single natural silk fibers, Biomacromolecules, 2011, 12, 3344-3349. 
54 T. Roman, W. A. Diño, H. Nakanishi and H. Kasai, Amino acid adsorption on single-walled carbon nanotubes, Eur. Phys. J. D, 2006, 1(38), 117-120.

55 C. Chen, R. Ran, Z. Yang, R. Lv, W. Shen, F. Kang, et al., An efficient flexible electrochemical glucose sensor based on carbon nanotubes/carbonized silk fabrics decorated with Pt microspheres, Sens. Actuators, B, 2018, (256), 63-70.

56 G. R. Plaza, J. Pérez-Rigueiro, C. Riekel, G. B. Perea, F. AgullóRueda, M. Burghammer, et al., Relationship between microstructure and mechanical properties in spider silk fibers: identification of two regimes in the microstructural changes, Soft Matter, 2012, 22(8), 615-626.

57 L. F. Drummy, D. M. Phillips, M. O. Stone, B. L. Farmer and R. R. Naik, Thermally Induced $\alpha$-Helix to $\beta$-Sheet Transition in Regenerated Silk Fibers and Films, Biomacromolecules, 2005, 6(6), 3328-3333.

58 N. Du, X. Y. Liu, J. Narayanan, L. Li, M. L. M. Lim and D. Li, Design of Superior Spider Silk: From Nanostructure to Mechanical Properties, Biophys. J., 2006, 12(91), 4528-4535.
59 S. Y. Cho, Y. S. Yun, S. Lee, D. Jang, K. Park, J. K. Kim, et al., Carbonization of a stable $\beta$-sheet-rich silk protein into a pseudographitic pyroprotein, Nat. Commun., 2015, 6, 7145.

60 M. B. Vázquez-Santos, E. Geissler, K. László, J. Rouzaud, A. Martínez-Alonso and J. M. D. Tascón, Comparative XRD, Raman, and TEM Study on Graphitization of PBO-Derived Carbon Fibers, J. Phys. Chem. C, 2011, 1(116), 257-268.

61 S. Lu, M. Jin, Y. Zhang, Y. Niu, J. Gao and C. M. Li, Chemically Exfoliating Biomass into a Graphene-like Porous Active Carbon with Rational Pore Structure, Good Conductivity, and Large Surface Area for High-Performance Supercapacitors, Adv. Energy Mater., 2017, (1702545), 1-9.

62 A. Jorio and A. Filho, Raman Studies of Carbon Nanostructures, Annu. Rev. Mater. Res., 2016, 46, 357-382.

63 C. Wang, K. Xia, M. Jian, H. Wang and M. Zhang, Carbonized silk georgette as an ultrasensitive wearable strain sensor for full-range human activity monitoring, J. Mater. Chem. C, 2017, 5, 7604-7611.

64 J. J. Boland, Flexible electronics: within touch of artificial skin, Nat. Mater., 2010, 10(9), 790. 